\title{
Individual-Based Spatially- Explicit Model of an Herbivore and Its \\ Resource: The Effect of Habitat Reduction and Fragmentation
}

Tanya Kostova, Tina Carlsen, and Jim Kercher

This article was submitted to

U.S. Department of Energy

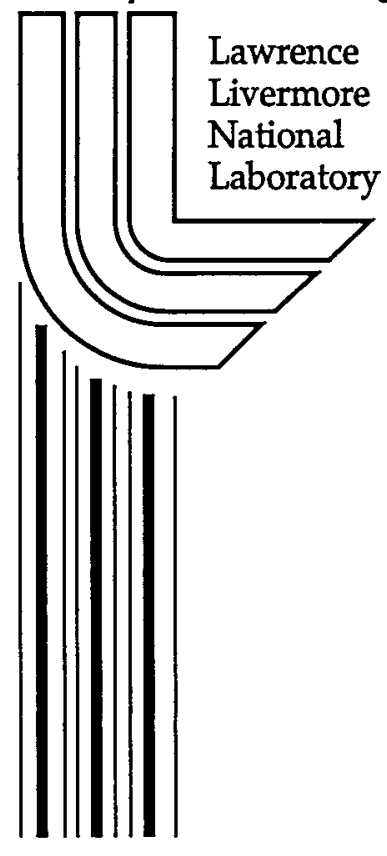

Conference of Mathematical Modelling of Population Dynamics, B'dlewo, Poland, June 24-28, 2002

June 17, 2002 


\section{DISCLAIMER}

This document was prepared as an account of work sponsored by an agency of the United States Government. Neither the United States Government nor the University of California nor any of their employees, makes any warranty, express or implied, or assumes any legal liability or responsibility for the accuracy, completeness, or usefulness of any information, apparatus, product, or process disclosed, or represents that its use would not infringe privately owned rights. Reference herein to any specific commercial product, process, or service by trade name, trademark, manufacturer, or otherwise, does not necessarily constitute or imply its endorsement, recommendation, or favoring by the United States Government or the University of California. The views and opinions of authors expressed herein do not necessarily state or reflect those of the United States Government or the University of California, and shall not be used for advertising or product endorsement purposes.

This is a preprint of a paper intended for publication in a journal or proceedings. Since changes may be made before publication, this preprint is made available with the understanding that it will not be cited or reproduced without the permission of the author.

This report has been reproduced directly from the best available copy.

Available electronically at http://www.doc.gov/bridge

Available for a processing fee to U.S. Department of Energy

And its contractors in paper from

U.S. Department of Energy

Office of Scientific and Technical Information

P.O. Box 62

Oak Ridge, TN 37831-0062

Telephone: (865) 576-8401

Facsimile: (865) 576-5728

E-mail: reports@adonis.osti.gov

Available for the sale to the public from

U.S. Department of Commerce

National Technical Information Service

5285 Port Royal Road

Springfield, VA 22161

Telephone: (800) 553-6847

Facsimile: (703) 605-6900

E-mail: orders@ntis.fedworld.gov

Online ordering: http://www.ntis.gov/ordering.htm

OR

Lawrence Livermore National Laboratory

Technical Information Department's Digital Library

http://www.llnl.gov/tid/Library.html 
Individual-Based Spatially-Explicit Model of an Herbivore and Its Resource: The Effect of Habitat Reduction and

\author{
Fragmentation
}

\author{
Tanya Kostova, Tina Carlsen and Jim Kercher \\ Lawrence Livermore National Laboratory \\ L-550, Livermore, CA 94550, USA
}

June 17, 2002

AMS Subject Classification: 92 
Author to whom correspondence should be sent: Tanya Kostova

L-550

Lawrence Livermore National Laboratory

7000 East Avenue,

Livermore, CA 94550, USA 


\begin{abstract}
We present an individual-based, spatially- explicit model of the dynamics of a small mammal and its resource. The life histories of each individual animal are modeled separately. The individuals can have the status of residents or wanderers and belong to behaviorally differing groups of juveniles or adults and males or females. Their territory defending and monogamous behavior is taken into consideration. The resource, green vegetation, grows depending on seasonal climatic characteristics and is diminished due to the herbivore's grazing. Other specifics such as a varying personal energetic level due to feeding and starvation of the individuals, mating preferences, avoidance of competitors, dispersal of juveniles, as a result of site overgrazing, etc. are included in the model. We determined model parameters from real data for the species Microtus ochrogaster (prairie vole). The simulations are done for a case of an enclosed habitat without predators or other species competitors. The goal of the study is to find the relation between size of habitat and population persistence. The experiments with the model show the populations go extinct due to severe overgrazing, but that the length of population persistence depends on the area of the habitat as well as on the presence of fragmentation. Additionally, the total population size of the vole population obtained during the simulations exhibits yearly fluctuations as well as multi-yearly peaks of fluctuations. This dynamics is similar to the one observed in prairie vole field studies.
\end{abstract}

Keywords:complexity, population dynamics, self-organization, population waves, individual-based models 


\section{Introduction}

The goal of this work is to study the effect of habitat size and the role of fragmentation on the population dynamics of a herbivore species. A herbivore interacts with its spatial environment through the resource it utilizes, i.e. by reducing the density of vegetation, transforming it into energy, and meeting its energetic needs to survive. Habitat size is affected and habitat fragmentation occurs as a result of human activities such as petroleum exploration and development. The reduction of habitat size leads to lower population sizes but may lead to increased population densities and overutilization of the resource and possibly to extinction of the population. The effect of fragmentation of various types is not clear $[11,3,13,12]$.

Biological systems are made up of systems themselves. Interacting biological entities such as animals, bacteria, etc. require the use of suitable modeling tools that take into consideration the heterogeneities of the individual entities and their environment and are able to incorporate both deterministic and stochastic effects. Differential equations deal with smooth quantities rather than aggregates of individuals and are not immediately suitable to adress the above requirements. The most suitable available now modeling technique is individual - based simulations.

We have constructed an individual - based model of herbivore-environment interaction which we parametrized for the species Microtus ochrogaster (prairie vole). We based the rules and numeric data of the model on a variety of literary sources. We have taken into consideration the climatic dependence of both vegetation growth and mammal breeding and define the local rules of movement of each individual to be driven by presence of resource and avoidance of competitors of the same gender. The spatial environment is represented as consisting of square cells with the size of the home range of $M$. ochrogaster. The population is subdivided into classes of wanderers and residents of spatial cells, which, together with the avoidance of same-sex competitors, models territorial behavior, a feature common to many species including the prairie vole. The female residents produce offspring if some additional conditions are met (energetic needs covered, enough generation time lap, presence of a male in the same cell). Individuals consume resource which replenishes itself due to growth dependent on precipitation and temperature.

The density of population time series produced as an output of the simulations resemble the ones observed in various vole population studies. It is a long-time puzzle ...

The emergent dynamics of the population in an enclosed habitat occurring as a result of the locally heterogeneous interactions between herbivores and resource has an interesting wave-like behavior in some intervals of time, while in others the patterns appearing as the population waves coalesce and disappear, are very irregular.

The effect of habitat area reduction was studied and showed that on smaller areas the population goes extinct for a shorter period of time than on larger ones. The simulations we did show that reducing habitat size reduces the time to extinction. A different set of simulations with removing patches of the habitat show that habitat fragmentation can have, in fact, a beneficial effect on the persistence of a population by reducing the amplitude of the fluctuations.

This paper is an attempt to bridge a gap between the ecological and mathematical approach to an individual- based model. We recognize that there is no existing theory of individual-based models. At the same time this approach is very appropriate for modelling complex systems and especially ones with spatial dependence.

The structure of the paper is as follows. In Section 2 we give an abstract formulation of an individual-based model as a nonlinear map. In Section 3 we summarize some of the population data we have gathered from the ecological literature. Section 4 is dedicated to a formal description of the model. We have made an attempt to write the mapping functions in a mathematical form and then briefly describe them in common language as is usually done in the ecological literature. In section 5 we describe the results of some simulations. 


\section{Individual-based models as generalized discrete dynamical systems}

Individual-based models (IBM) are discrete computational simulations which explicitly model large numbers of individual, heterogeneous entities interacting with each other and their environment.

IBM are the only available modelling approach that can meet the needs of a fast growing number of biological applications characterized by processes that incorporate entities with a large number of possible states changing in time or space due a large number of possible mechanisms. The simulations make it possible for the scientist to carry out virtual experiments and test hypotheses based on available experimental and empirical data.

The IBM approach has certain advantages in comparison with partial differential equations models. They are summarized as follows.

a) IBM are by definition a natural tool for modelling systems made of systems. Each individual is represented as a system of interacting components such as age, energetic budgets, location, status, etc. and these individual systems interact with each other in ways depending on their own state and the state of the environment.

b) Possibility to work with a large amount of independent variables. In IBM the variable attributes of the entities play the same role as the independent variables (time, spatial coordinates, etc.) in PDEs. Solving PDEs with more than 4 variables $(t, x, y, z)$ is a challenging task even today. IBM can incorporate dozens of independent variables and make the models much more realistic.

c) Straight-forward model formulation. Models are based on rules observed in the real system to be simulated and are readily understandable by life scientists.

d) Versatility. IBM give an easy way to incorporate both deterministic and stochastic components in the model.

e) The non-averaged behavior of the output allows for observing phenomena that would be impossible with PDEs but closer to reality. Shnerb et al, [37] find that in conditions in which the continuum approach would predict the extinction of all the population, the slightest microscopic granularity insures the emergence of macroscopic localized sub-populations with collective adaptive properties which allow their survival and development. This is observed in our simulations as well.

IBM are getting increasing popularity in the ecological community $([2,6,21,26,29,33,38,40$, $41]$ ), but are readily extended to a finer scale (microbial communities [25, 20]) or a slightly different setting (epidemic simulations [14]).

In an attempt to present in a formal language the essence of an IBM, we can describe it in mathematical terms as a discrete-time highly-dimensional nonlinear map.

IBM resemble discrete dynamical systems which have a relatively well developed theoretical foundation. However, IBM are a more general type of a map than dynamical systems and therefore, the theoretical framework for these is still to appear. In a discrete dynamical system $\mathbf{x}(\mathbf{n}+\mathbf{1})=$ $\mathcal{F}\left(\mathbf{x}(\mathbf{n})\right.$ ), where $\mathbf{x}(\mathbf{i}) \in \mathbf{R}^{\mathbf{m}}$, the dimensions of the prototype and the image are the same and the map $\mathcal{F}$ is a deterministic (usually) continuous relation.

An IBM is a nonlinear map which transforms a certain amount of matrices of $\mathcal{M}$ of certain (high) dimensions $N_{i}(n) \times m_{i}$ into (the same amount of) matrices whose dimensions are usually different.

The matrices are formed by the vector individuals. More specifically, at time $t=n$ there are $N_{i}(n)$ individuals, $i=1, \ldots, l$ of $l$ different types. The individuals are vectors $A_{j}^{i}$ whose components are the individual data:

$$
A_{j}^{i}=\left(\operatorname{data}_{1}^{i}, \ldots ., \operatorname{data}_{m}^{i}\right)^{T}, i=1, \ldots, N_{i} .
$$

At time $t=n+1$ a map $\Phi(n)$ converts $M_{i}(n)$ into $M_{i}(n+1)$ which is a matrix of dimensions $N_{i}(n+1) \times m_{i}$. These dimensions can be different from $N_{i}(n) \times m_{i}$.

$$
\Phi(n): M_{i}(n) \longrightarrow M_{i}(n+1) .
$$


The matrices' dimensions vary since the number of entities at each time step is variable (are born or die at various moments). This differentiates IBM from discrete dynamical systems, as they have a fixed number of equations across time. Additionally, the map $\Phi$ can contain random parameters or be entirely deterministic.

If no random parameters are present, and the nature of the problem is such that all matrices $M_{i}(n), i=1, \ldots, l ; n=0,1, \ldots$ are contained in some finite dimensional space (i.e. $N_{i}(n)$ and $m_{i}$ are bounded from above for all $n$ ), one could put the IBM into the form of a discrete dynamical system and hopefully apply some of the existing theory ([23]). One could expect the existence of attractors and thus, see the formation of specific patterns governing the dynamics. In the model and simulations presented in this paper we do observe the spontaneous formation of wave-like patterns and phenomena resembling turbulence.

\section{The prairie vole: experimental and empirical data}

Some of the vole data were collected through surveys with no experimental manipulation. We call it empirical data. Data collected through enclosure and removal experiments are actual "experimental" data.

Demographic and physiological data. The data on life expectancy of the prairie vole is very inconsistent because the measurements depend on predation at the specific region as well as climatic factors. The prairie vole mean life expectancy was established by Getz et al. [18] to be between 50 and 80 days depending on the season. Animals born in summer and autumn had higher life expectancy (61-80 days) than ones born in winter and spring (41-50 days). However, some voles born in the summer or autumn season live up to 450 days, while these born in the winter or spring live up to 120-150 days [18].

Voles are considered adults if they have reached 30 days of age or approximately $30 \mathrm{~g}$ weight.

An adult prairie vole produces in average 3-5 offspring per litter and 3-4 litters per year. The gestation period is 21 days and is approximately equal to the generation time (period between pregnancies ), [16, 34].

The daily food intake depends on the quality of the food but for green (undried) grass it can be estimated as follows. Sawicka-Kapusta et al [36] estimates for the caloric value of biomass of various fodders, for grass being $0.85-0.98 \mathrm{kcal} / \mathrm{g}$ biomass. Bradley [5] calculates the daily food intake of $M$. ochrogaster to be approximately $600 \mathrm{cal} / \mathrm{g}$ body weight. For $30 \mathrm{~g}$ body weight then the daily caloric requirement is $18 \mathrm{kcal}$. Using the data from Sawicka-Kapusta et al. [36] we obtain that the dailly grass intake for an adult vole will be about $20 \mathrm{~g}$.

Climatic dependence. Breeding shows seasonal dependence and is defined as between February and November. Rose and Gaines [34] report no breeding in December and January and even through April in one of the years, while Krebs et al [24] report very low percentage of breeding females in November- January and peaks of breeding in August, September and October. Keeping in mind the bioenergetics of these small mammals, which spend most of the produced metabolic energy for maintaining their body temperature, these reports are reasonable.

The home range. Species that exhibit site fidelity have a mean amount of territory that they move around daily and defend, the home range(HR). HR size has been demonstrated to be related to the energetic needs of the mammal, [28]. Thus, it is gender and season dependent. The HR size has been reported to be somewhere between 10 and $50 \mathrm{~m},[1,15,39]$.

Factors governing population density. It is known from observations [18], [24] that highest vole population densities occur in the late autumn (October, November), that densities decrease drastically in January-March, the lowest densities usually occurring in March and then start steadily increasing until late autumn. 
The population density of the prairie vole depends on the quality and quantity of vegetation as well as on the presence of sympatric herbivore species and/or predators. Food quality is very important for the population dynamics of the prairie vole. For example, alfalfa has a higher nutrition value than tall grass [8]. The maximum vole density in tallgrass prairie as recorded in [17] was 90/ha while the mean density was $7 /$ ha showing high variability during a 25 year study. Cole et al [8] report maximum density $38 / \mathrm{ha}$ and $10 / \mathrm{ha}$ respectively in a next year for the above mentioned types of vegetation.

Experiments on enclosed grids show that dispersal plays an important role in population density regulation. Density can become quite high in enclosed areas. Krebs et al [24] compared fenced and unfenced grids and observed a rapid increase in population density in the fenced case, which led to overgrazing. A very interesting experiment of introducing 18 prairie voles in an empty fenced plot of $0.8 \mathrm{ha}$ led to an extreme density of 494 vole/ha (395 voles in the plot) after 7 months. Consequently the vegetation was overgrazed and the population fell to 11 voles in early April when it started to increase steadily.

Absence or presence of a predator also plays an important role on population density regulation. Desy and Batzli [10] conducted experiments on enclosed plots with mixed vegetation including blue grass and observed maximum density of $550 /$ ha on enclosures with no predators and added food, while in enclosures with no supplemented food and with predators, the density was stable at about $90 /$ ha.

Microtine species, one of which is the prairie vole species, exhibit seasonal and multi-annual population size fluctuations. The physiology of the vole, a very small mammal, which needs an enormous amount of energy (relative to its body mass) to maintain constant body temperature, is clearly seasonally dependent. At low temperatures, the vole has to diminish or abandon certain highenergy-consuming activities in order to survive. Breeding (as seen above), deaths due to predation and life expectancy are seasonally dependent phenomena. Several authors have reported multiannual fluctuations in population density with occurring peaks with frequency $2-4$ years. Rose and Gaines [34] note that microtine cycles vary from 2 to 5 years in duration, with many having 3-4 year intervals between peaks. Getz and Hofmann [17] report high-peak densities appearing with periods of 4.3 years and secondary peaks at 2.1-2.4 years on three different habitats and that these fluctuations are synchronized for the three different habitats. The periodicity of the fluctuations does not depend on the external manipulations of the habitat (burning of tall grass, mowing of blue grass and alfalfa) but their amplitude does depend on the quality of food (alfalfa has the highest caloric quality, tall grass the lowest) and are positively correlated with it [19]. Cole and Batzli [8] also note that food availability may influence the amplitude of fluctuations but not the periodicity of the cycle. The multi-annual population size oscillations of the microtine species is a continuing puzzle to ecologists.

There are various hypotheses and studies related to them concerning vole population fluctuations. Some authors study the connection of population density and juvenile survival (Krebs et al [24], Getz et al [18]), while others put forward as a possible cause changes of food quality, [8].

Social relations. Getz and McGuire [16] show that the majority of single males are wanderers while a considerably smaller proportion of single females wander. Wanderers frequently visit the nests of pairs but are kept away by the male [27]. Unrelated adults join communal groups only if they include already grown offspring male [27]. New pairs were formed after the winter period from surviving individuals mainly from different social groups [16]. Most of the new pairs formed in summer-autumn were made from two wandering individuals.

Prairie voles are monogamous animals. Paired males and females cohabit a nest and a home range, [16]. McGuire and Getz, [27] find that paired males exclude nonresident males from the areas around their nests. This was also confirmed in [16].

Territory specificity and defense. There is little overlap in use of space by social groups even at high population densities, [27]. Spatial behavior limits interaction between members of adjacent 
social groups. Jacquot [22] found that vacant territories were fast occupied. Density of population was positively correlated with the time necessary to emigrate. Female prairie voles immigrated in response to vacant territories and not to potential mates. Males also immigrated in response to vacant territories. It is generally accepted that males show preference to territories with present females.

\section{Description of the model}

\subsection{The variable matrices}

At each time moment $n$ the total amount of present animals is $N_{1}(n)$. The animal objects

$$
A_{1}(n), \ldots ., A_{N_{1}(n)}(n)
$$

possess the following $m=9$ variable data: coordinates of the current location of the animal nrow and $n c o l$; age age, gender gen (this is a variable because animal $A_{k}(n)$ and animal $A_{k}(l)$ are in general different objects at different times $n$ and $l$ ) where gen $=1$ if the animal is male and 2 if female; status stat (which can be wanderer or resident); time since last pregnancy $\tau$ (defined as 0 for males and a positive value for females); energetic budget enbudget: a variable reflecting the energetic status of the animal and important for its survival. Finally, each animal object is given a variable remove (defined as 0 or 1 ), which indicates whether the animal is dead (remove $=1$ ) and will be removed from the matrix and a variable birth indicating whether it gives offspring at time $n$ $($ birth $=1)$ or not $($ birth $=0)$.

Thus, at any time moment $n$ the animal objects with their data represent a $N_{1}(n) \times 9$ matrix, the matrix of animal objects.

$$
\mathcal{A}=\left(\begin{array}{lccr}
\text { nrow }_{1} & \text { nrow }_{2} & \ldots & \text { nrow }_{N_{1}} \\
\text { ncol }_{1} & \text { ncol }_{2} & \ldots & \text { ncol }_{N_{1}} \\
\text { age }_{1} & \text { age }_{2} & \ldots & \text { age }_{N_{1}} \\
\text { gen }_{1} & \text { gen }_{2} & \ldots & \text { gen }_{N_{1}} \\
\text { stat }_{1} & \text { stat }_{2} & \ldots & \text { stat }_{N_{1}} \\
\tau_{1} & \tau_{2} & \ldots & \tau_{N_{1}} \\
\text { enbudget }_{1} & \text { enbudget }_{2} & \ldots & \text { enbudget }_{N_{1}} \\
\text { remove }_{1} & \text { remove }_{2} & \ldots & \text { remove }_{N_{1}} \\
\text { birth }_{1} & \text { birth }_{2} & \ldots & \text { birth }_{N_{1}}
\end{array}\right)
$$

$N_{1}(n)$ can take very big values depending on the amount of spatial cells (area size) we consider. In a typical simulation $N_{1}$ reaches several hundred thousands up to a million. The lower bound is naturally 0 , but in general, even if $N_{1}$ does not become so small, it is highly variable, falling to several thousands in the winter months.

All animal objects share constant data characteristic of the species: average daily food intake $D F I$, life expectancy $L E$, maturation age $M A$, generation time $G T$, litter size $L S$. The values of these quantities are taken to be in the ranges outlined in the previous section. Additionally, the following controlling parameters relevant for the energetic budget enbudget are introduced: $M E B$, maximum energetic budget, $M e a n E B$, mean energetic budget, $J E B$, juvenile energetic budget. We explain the meaning of the controlling parameters in a following subsection.

Space is represented as a collection of a constant number $N_{2}$ of square cells, $C_{1}, \ldots C_{N_{2}}$, each one with an area roughly equal to the home range of the herbivore. The location of each cell is represented by two coordinates (row and column). The cells are individual objects possessing the following 5 variables: quantity of vegetation veg in the cell, total number pop of animals in the cell 
(juveniles, male and female), number of present male herbivores mpop, female herbivores fpop, and a variable $p$ indicating whether the spatial cell has been polluted and therefore should be removed (i.e. it has been developed, polluted or otherwise rendered uninhabitable). Thus, at any time moment $n$ the cells with their data represent a $N_{2} \times 5$ matrix, the matrix of cell objects.

$$
\mathcal{C}=\left(\begin{array}{lccr}
\operatorname{veg}_{1} & \operatorname{veg}_{2} & \ldots & \operatorname{veg}_{N_{2}} \\
\text { pop }_{1} & \text { pop }_{2} & \ldots & \text { pop }_{N_{2}} \\
\text { mpop }_{1} & m p o p_{2} & \ldots & \text { mpop }_{N_{2}} \\
\text { fpop }_{1} & \text { fpop }_{2} & \ldots & \text { fpop }_{N_{2}} \\
p_{1} & p_{2} & \ldots & p_{N_{2}}
\end{array}\right)
$$

Note that we address the spatial cells in two different ways: we order the cells sequentially as $C_{1}, \ldots C_{N_{2}}$, and we also address them by using their coordinates when defining an animal object. We need to have a way to link the coordinates with the cell.

Thus, given row and column coordinates, nrow, ncol, let \#(nrow, ncol) be a function whose value is the sequential number of the cell with coordinates nrow, ncol. Otherwise said, $C_{\#(\text { nrow,ncol) }}$ has coordinates $(n r o w, n c o l)$. An animal object $A_{j}$ will then be located in the cell $C_{\#\left(n r o w_{j}, n c o l_{j}\right)}$.

The dimensions of the cell objects matrix do not change over time. The number of columns of the animal objects matrix may change at each time step because of births and deaths. The newborn objects are added as last columns of the matrix, while the dead objects (with remove $=1$ ) are deleted and the list of animals is updated. Thus, at time $n$ an animal may have index $j$ in the list, while at time $n+1$ this index may be different.

\subsection{The mapping functions}

The time unit used in the model is 1 day. We simplify the simulations by assuming that a month has 30 days and an year has 360 days.

The mapping functions describe the transition from matrices $\mathcal{A}(n)$ and $\mathcal{C}(n)$ to $\mathcal{A}(n+1)$ and $\mathcal{C}(n+1)$. The order in which the maps are executed is important and we are keeping to it in the following description.

In this model the only random map is the one that controls the animals' movements between cells.

(0) If at time $n$ animal $A_{j}$ has index $j$ and remove ref $_{j}=0$, then we define $i m g(j)$ as the function which calculates the new index, at time $n+1$, of animal $j$. Keeping in mind the way we do the rearrangement of the list, we find that $i m g(j)=j$ - the number of animals that died and had index less than $j=i m g(j)=j-\sum_{k<j}$ remove $_{k}$.

(1) Cell population numbers

$$
\operatorname{pop}_{j}(n+1)=\sum_{i=1}^{N_{1}(n+1)} \delta_{0}\left(\#\left(\text { nrow }_{i}, n \operatorname{col}_{i}\right)-j\right)
$$

where $\delta_{a}(x)$ is the delta function: $\delta_{a}(a)=1,0$ otherwise.

$$
\begin{gathered}
\operatorname{mpop}_{j}(n+1)=\sum_{i=1}^{N_{1}(n+1)} \delta_{0}\left(\#\left(\text { nrow }_{i}, n c o l_{i}\right)-j\right) \delta_{1}\left(\text { gen }_{i}\right) \\
\text { fpop }_{j}(n+1)=\sum_{i=1}^{N_{1}(n+1)} \delta_{0}\left(\#\left(\text { nrow }_{i}, \text { ncol }_{i}\right)-j\right) \delta_{2}\left(\text { gen }_{i}\right)
\end{gathered}
$$


(2) Aging

This is the simplest mapping from time $n$ to time $n+1$.

$$
a g e_{i m g(j)}(n+1)=a g e_{j}(n)+1 .
$$

(3) The energetic budgets

To model the effect of food and starvation on survival, we use the variables $\operatorname{enbudget}_{j}(n), j=$ $1, \ldots, N_{1}(n)$.

$\operatorname{enbudget}_{i m g(j)}(n+1)=\operatorname{enbudget}(n)-1$, if $\operatorname{veg}_{\#\left(\operatorname{nrow}_{j}(n), n c o l_{j}(n)\right)}<D F I \times \operatorname{pop}_{\#\left(\operatorname{nrow}_{j}(n), n c o l_{j}(n)\right)}$

Otherwise,

$$
\text { enbudget }_{i m g(j)}(n+1)=\text { enbudget }(n)+1,
$$

if $a g e_{i m g(j)} \geq M A$ and enbudget $(n)<M E B$ or if age $i_{i m g(j)}<M A$ and enbudget $(n)<J E B$.

That is, if the animal feeds, it increases its energy store (unless it is at the maximum) and if it cannot feed because of lack of enough resource, its energy store drops. Additionally, there is a limit to the energy an animal can store, which is smaller for the juveniles.

(4) Survival or removal

remove $_{j}(n+1)=0$ if age $e_{j}(n+1)<L E$ and if enbudget $(n+1)>0$. Otherwise, remove $_{j}(n+1)=$ 1. That is, an animal dies if its energy budget became 0 or if its age reached the life expectancy.

(5) Status change

The variable stat $_{j}$ changes its value as follows.

$\operatorname{stat}_{i m g(j)}(n+1)=$ resident, if $\operatorname{age}_{i m g(j)}(n+1)<M A$.

$\operatorname{stat}_{i m g(j)}(n+1)=$ wanderer, if $\operatorname{age} e_{i m g(j)}(n+1)=M A$.

If $a g e_{i m g(j)}(n+1)>M A$ and if $\operatorname{stat}_{j}(n)=$ wanderer, then

$$
\operatorname{stat}_{i m g(j)}(n+1)=\text { resident, }
$$

if $\operatorname{gen}_{j}(n)=1$ (male) and $m p o p_{\#\left(n r o w_{i m g(j)}(n+1), n c o l_{i m g(j)}(n+1)\right)} \leq 1$

or if $g_{e n}(n)=2$ (female) and fpop \#(nrow $\left.n_{i m g(j)}(n+1), n c o l_{i m g(j)}(n+1)\right) \leq 1$.

Otherwise, stat $_{i m g(j)}(n+1)=$ wanderer.

If $\operatorname{age}_{i m g(j)}(n+1)>M A$ and if $\operatorname{stat}_{j}(n)=$ resident, then

$\operatorname{stat}_{i m g(j)}(n+1)=$ wanderer if $\operatorname{veg}\left(\#\left(\operatorname{nrow}_{i m g(j)}(n), \operatorname{ncol}_{i m g(j)}(n)\right)=0\right.$. Otherwise $\operatorname{stat}_{i m g(j)}(n+$ 1) $=$ resident.

In plain words, all juveniles are residents until they reach adult age, when they become wanderers. Adults who were wanderers can become residents if they find themselves in a cell with no other individuals of the same gender, otherwise they continue to wander. Thus, each cell can contain only one pair of adult residents (plus their immediate offspring) and many wanderers. Adult residents become wanderers if the cell gets overgrazed and has no vegetation left.

Wanderers do not contribute to the population growth but use the resource, thus controlling the population in certain bounds. 
(6) Births

$$
\begin{aligned}
& \operatorname{birth}_{j}(n)=0 \text { if } \bmod _{360}(n)>300 \text { or }<30 \\
& \text { or if } \text { remove }_{j}(n) \neq 0 \\
& \text { or if } \text { gender }_{j}(n) \neq 2 \\
& \text { or if } a g e_{j}(n) \leq M A \\
& \text { or if } s_{t a t}(n)=\text { wanderer } \\
& \text { or if } \operatorname{mpop}_{\#\left(\operatorname{nrow}_{j}(n), n c o l_{j}(n)\right)}=0 \\
& \text { or if enbudget } t_{j}<M e a n E B \text {; } \\
& 1 \text {, otherwise. }
\end{aligned}
$$

In other words, a female vole gives birth only in the months between February and October and if it is an adult resident, with energetic budget not less than $M e a n E B$ and if a male animal is present in the cell.

(7) The time between pregnancies $\tau$.

$$
\begin{gathered}
\tau_{i m g(j)}(n+1)=\tau_{j}(n)+1 \text { if } b i r t h_{i m g(j)}(n+1)=0 \\
0, \text { otherwise. }
\end{gathered}
$$

\section{(8) Location change}

Wanderers change their location at each time moment until (if) they become residents.

If $\left(\operatorname{nrow}_{j}(n), \operatorname{ncol}_{j}(n)\right)$ are the coordinates of the location of animal $A_{j}$ at time $n$, then the 8 surrounding cells have pairs of coordinates $\left(\right.$ nrow $\left._{j}-1, n_{\text {col }}-1\right), \ldots,\left(\right.$ nrow $\left._{j}+1, n \operatorname{col}_{j}+1\right)$.

Let $C_{\#\left(\text { nrow }_{j}-1, \text { ncol }_{j}-1\right)}, \ldots, C_{\#\left(\text { nrow }_{j}+1, n c o l_{j}+1\right)}$ be the 8 neighboring cells. To ease notation, let us denote them by $C_{j(1)}, \ldots, C_{j(8)}$.

If $\operatorname{stat}_{j}(n)=$ resident, then

$$
\operatorname{nrow}_{i m g(j)}(n+1)=\operatorname{nrow}_{j}(n), \operatorname{ncol}_{i m g(j)}(n+1)=n \operatorname{col}_{j}(n) ;
$$

if $\operatorname{stat}_{j}(n)=$ wanderer, then $\left(\right.$ rrow $\left._{i m g(j)}(n+1), \operatorname{ncol}_{i m g(j)}(n+1)\right)$ takes randomly one of those pair values $\left(\right.$ nrow $_{j}-1$, col $\left._{j}-1\right), \ldots,\left(\right.$ nrow $\left._{j}+1, n_{\text {col }}+1\right)$ for which $v e g_{j(k)}>0$. That is, when an animal needs to change its location, it chooses in a random manner to move in one of the directions with available food resource.

(9) Vegetation growth and depletion

The variables $\operatorname{veg}_{i}(n), i=1, \ldots, N_{2}$ are transformed into veg $_{i}(n+1), i=1, \ldots, N_{2}$ as follows

$$
\operatorname{veg}_{i}(n+1)=\operatorname{veg}_{i}(n)+r(n)-\operatorname{pop}_{i}(n) . D F I,
$$

where $r(n)$ is the change in the amount of green vegetation between days $n$ and $n+1$ due to growth or death (drying out, freezing, etc.) caused by climatic conditions. $r(n)$ is positive in time periods when the vegetation growth is larger than decay and negative otherwise. It is a forcing function similar to the ones used in continuous population models with climatic forcing. We use an artificially generated 30 year time series of biomass (shown on Figure 1) to model the monthly amount of vegetation per square meter $\delta(k)$ in month $k$, granted the average temperature and precipitation data for this month are known. Assuming that the rate of vegetation growth does not depend on whether it is being grazed, that a month has 30 days and that vegetation grows with the same rate within a month, then

$$
r(n)=(\text { area of the cell }) \times\{\delta(k+1)-\delta(k)\} / 30,
$$


where $k=\left[\frac{n}{30}\right]$ and $\delta(0)$ is the initial per square meter amount of vegetation.

(10) Genders. Naturally,

$$
\operatorname{gen}_{i m g(j)}(n+1)=g e n_{j}(n) .
$$

(10) Finally, $p_{j}(n)$ is an externally defined function.

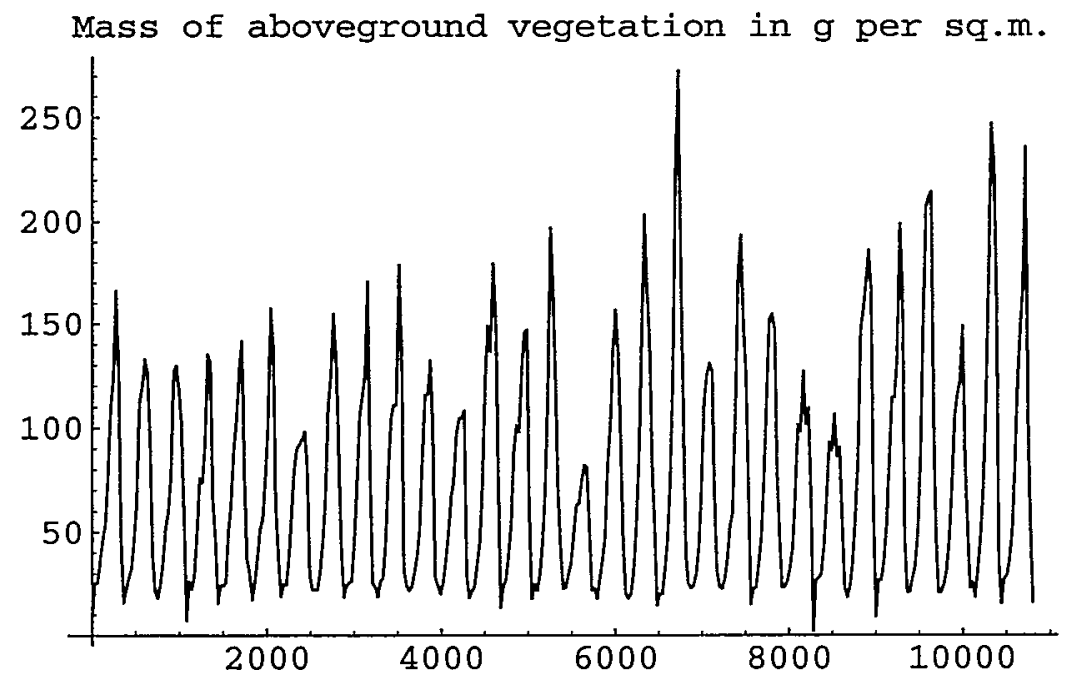

Figure 1. Plot of artificially generated green vegetation in $\mathrm{g} / \mathrm{sq} . \mathrm{m}$. Time is in days.

\section{Simulations}

All calculations simulate a "fenced plot", i.e. no animals are allowed to enter or leave the site.

\subsection{Parameter values and initial distributions}

In the simulations we use numeric values of the parameters close to the ones reported in the literature (compare with section 3). The area of each cell is 900 square meters ( $30 \mathrm{~m}$ by $30 \mathrm{~m}$ cells), roughly corresponding to the prairie vole's HR size. The initial time is set as January 1 1960. The life expectancy $\mathrm{LE}$ was varied accross simulations between 90 and 140 days (which is close to the maximum observed life span of voles born in winter). Additionally, $G T=21$ days, $M A=30$ days as described by real data. The litter size $L S$ is taken to be equal to 2 , a twice lower value than the number of newborns, to account for a high mortality of offspring reported in many publications. $D F I=10$ is also taken at a lower value than the one known for adult voles, because it is an average value for both adults and juveniles. The controlling energetic budget values were taken as $M E B=20 ; M e a n E B=15 ; J E B=2$. These are rather arbitrary because of lack of data. $M E B=20$ has the meaning that an adult vole would die after 10 days of starvation, while a juvenile would endure only 2 days of starvation. 
Initially all cells were assumed to contain the same amount of vegetation (no spatial heterogeneity was assumed).

\subsection{Results}

A legitimate question that arises is how to compare the simulation results. We use the term persistence in the sense of "time to extinction". Our simulations were done for a 30 year period of time. We cannot say if the populations that persisted for 30 years would go extinct in a longer period of time or if they will persist for ever. This is a very interesting question but unfortunately we do not have the theoretical framework that would allow us to find an answer.

General observations.

We did a variety of simulations with the model. The initial distribution were either randomly generated over the whole plot or were modelled as small area sources. All initial distributions consisted of wandering adults. The simulation results share some similarities as follows.

As expected, vole population densities (the total population divided by the area in hectares) fell drastically in the winter months due to overgrazing and to the lack of breeding. Populations reached maximum densities in the end of the autumn (usually in November) and minimum densities in February- March, similar to that reported in section 3.

The time series of the vole densities showed annual peaks (probably due to the seasonality of the vegetation data) and multi-annual fluctuations with peaks separated by periods ranging between 2 to 5 years, similar to what is described in section 6 . Figure $2 \mathrm{a}$ shows a typical time series of a $120 \times 120$ cells simulation with an initial distribution of 348 animals aggregated in a small amount of cells. Similar time series patterns are seen on Figure $4 \mathrm{~b}$ and Figure $3 \mathrm{~b}$ (the non-extinct case). Figure 4b shows an interesting observation: the peaks in density appear in the same years for the three plots. That is, the amplitude of fluctuations depends on the size of the plot but the periodicity does not. This is comparable to the mentioned in section 3 observations that the periodicity of fluctuations does not depend on the quality of the plot. Our simulations show that the peaks in population density do not coincide with the peaks in the resource density. One can hypothesise that the multi-annual fluctuations are due to the combined effect of the oscillatory vegetation forcing and the intrinsic population density oscillations due to the periodicity of breeding.

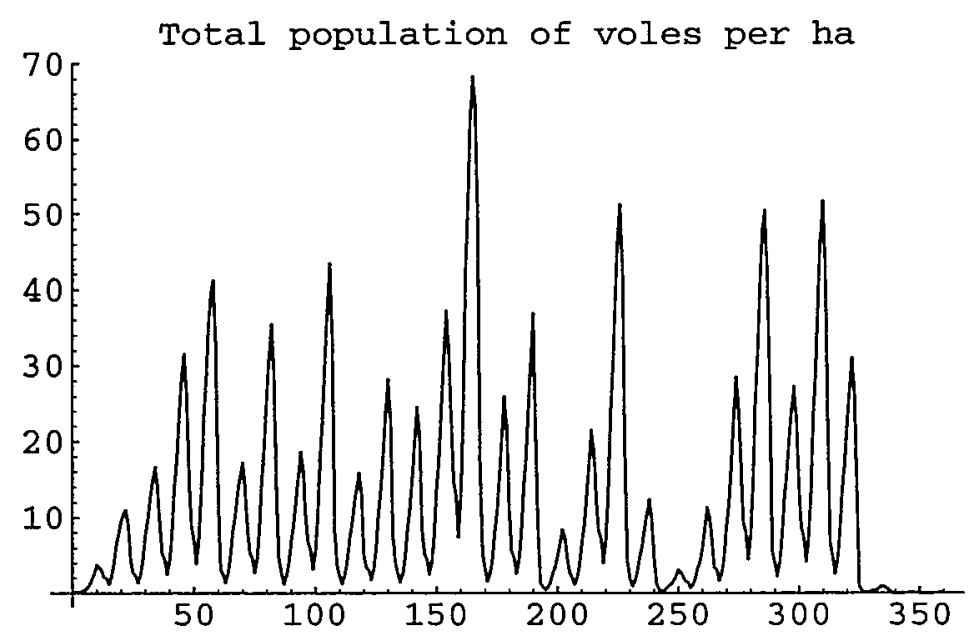


Figure 2a. Annual and multi-annual population density fluctuations.

See text for details.

Further, it is important to note that the maximum densities did not exceed 200 per ha, in the expected limits for this species in a closed area.

Specific spatial patterns similar to wave motion formed in all simulations. Simulations starting with uniform random distribution over the whole plot showed the formation of wave patterns after the first year when separate individuals survived and started reproducing and dispersing randomly. The random dispersal in all directions led to the formation of oval populated regions with highest density in the center of the ovals. The subsequent overgrazing led to the removal of animals from the central part of the ovals thus forming rings of nonzero density, which we refer to as "waves". The waves further expanded and their central parts were removed as results of overgarzing and deaths. The waves hit one another subsequently forming various patterns of irregular structure. Figure $2 \mathrm{~b}$ shows typical patterns illustrating this observation.

Figure 2b. Formation of wave - like patterns from a uniformly random distribution on a spatial plot with a removed central region. The top left graph corresponds to the initial distribution in January of year I, the second on top corresponds to December of year 1, the bottom left - to November of the second year and the bottom right - to December of the second year.

Optimal conditions for survival.

The survival of the population depended on the initial distribution and on the parameters we varied. If the initial distribution density was low (0-2 animals per cell) and especially if the inhabited cells were sparse, the population died out because of a low number of mating pairs formed and low amount of offspring throughout the breeding season. Additionally, if the initial density was high, the population amassed very high densities in the subsequent years, overgrazed the whole region and perished (compare with experimental results described in section 3, [24]). At high and low initial densities the persistence of the population showed high sensitivity to initial conditions in the sense that different initial distributions with similar total population numbers developed different outcomes; the population sometimes persihed after a few years, while in other cases it persisted for the 30 year test period. Overall, one can hypothesise the existence of an optimal range of initial 
population sizes that guarantee the persistence of the population.

The importance of area size.

In one simulation we compared 3 "fenced plots" of 200,300 and 400 cells respectively. We started the simulations with the same initial distribution of voles which was randomly generated in the smallest plot. The plots are contained into one another, with the bottom left corner being common for all plots. That is, the 300 plot was five ninths empty and the 400 plot was three quarters empty in the beginning. The parameters of the model were the same as described in the previous section with the life expectancy being 120 days. As an illustration, Figure 3a shows the density distributions in the largest plot in December of the second, tenth and fifteenth year.

Figure 3a. Spatial distribution of vole densities in a $400 \times 400$ plot in months $24,120,180$ (from left to right). See text.

The vole population on the smallest plot went extinct in about 180 months, on the second plot - in 269 months, while on the third plot it persisted during the whole simulation period of 360 months. Figure $3 \mathrm{~b}$ demonstrates the time series of the total population density per ha. The dashed line corresponds to population density on the largest plot, the dotted line - to the middle-sized plot and the continuous line - to the smallest plot. 


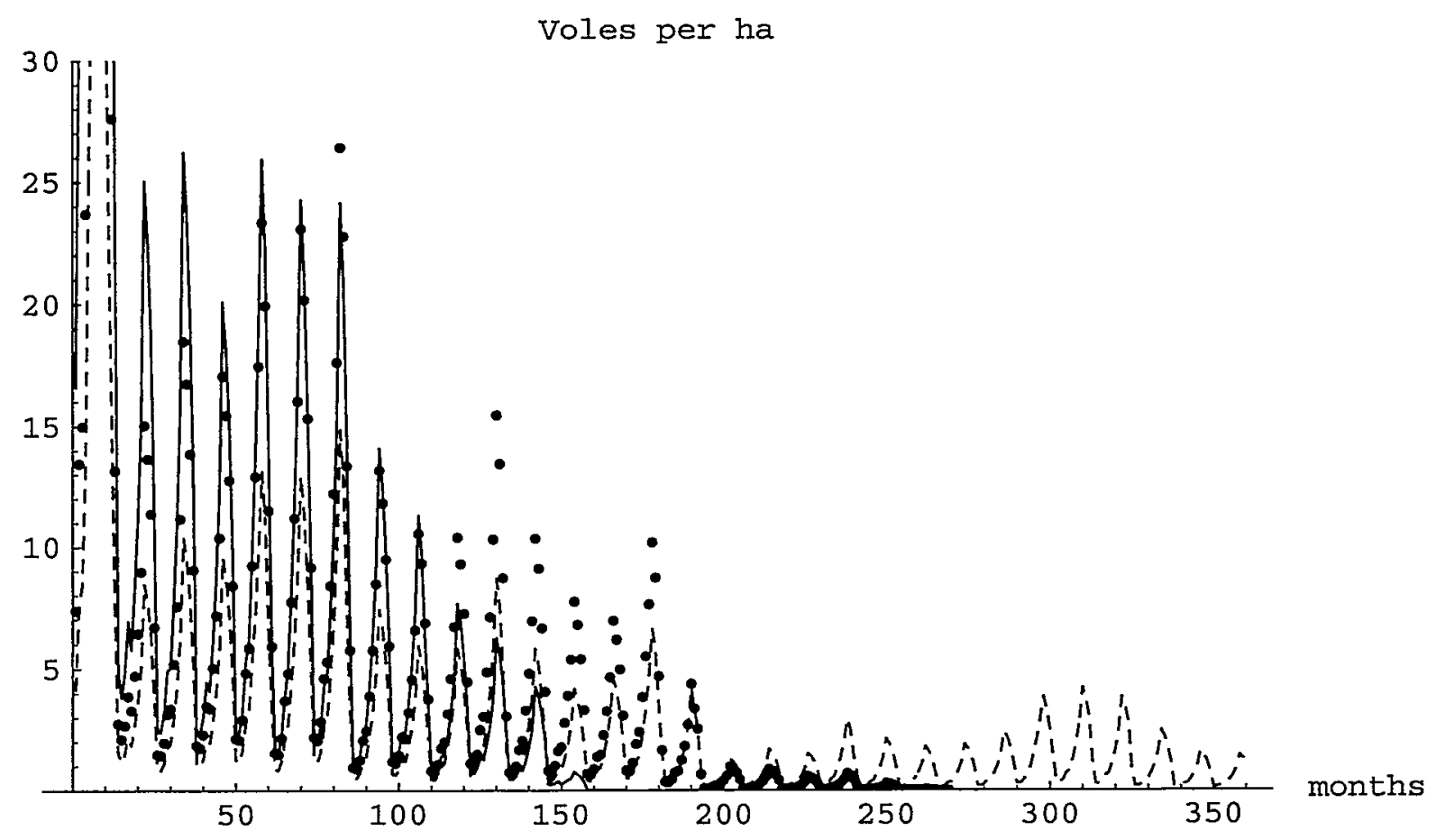

Figure 3b. Comparison of the length of population persistence on three plots of $3600 \mathrm{ha}, 8100 \mathrm{ha}$ and 14400 ha. See text for details.

The smaller the plot, the higher the maximum and average density per ha and the larger the standart deviation. The latter measures the instability caused by oscillations. The maximum densities for the $200 \times 200,300$ and 400 plots were 100, 82 and 46 correspondingly while the average densities were correspondingly $12,6,3$ and the standard deviations were $17 \%, 11 \%, 10 \%$, respectively.

On the smaller plots the population extinction is caused by severe overgrazing of most of the plot and subsequent starvation during the winter months. On the largest plot overgrazing does happen locally, while there are vast areas with sufficient vegetation remaining.

We did similar simulations with other sizes of "fenced plots" and other initial distributions and we observed similar monotonous dependence of area size and length of population persistence.

The importance of dispersal and predation.

Our simulations model a fenced habitat of a single population. The lack of dispersal outside the area causes the appearance of very high population densities and subsequent overgrazing. The insufficiency of vegetation combined with the lack of breeding in the winter months causes severe population crashes and may lead to rapid extinction. We already noted that extinction was dependent on area size, initial densities, life expectancy, etc. Any factor that contributes to lowering population density, such as dispersal through the boundaries of the habitat and predation should act in the direction of increasing the population's chance of survival.

Fragmentation is not necessarily a bad thing.

We compared the results of simulations on cells with various sizes with identical initial distributions but with various types of "obstacles". The obstacles are parts of the landscape unaccessible (or avoided) by the animals. Here we discuss the comparison between two simulations, one with- 
out obstacles and the other in the form of a "checkerboard landscape", see Figure 4a for a visual representation.

Figure 4a. Density distributions of voles in different points of time in a checkerboard habitat. The overall wave-like progression of density is preserved.

The initial distribution simulated an experiment in which 42 adult voles were released into the plot in its bottom left corner. Interestingly, the spatial wave-like progression of density distribution was preserved as seen on Figure 4a. The patchiness of the landscape decreased the amplitude between the lowest and highest population per ha densities. We compare three simulations with same initial distributions: on a $200 \times 200,144 \times 144$ and checkerboard plots. The $144 \times 144$ plot has almost the same area size as the total area of all accessible patches of the checkerboard plot. We compare these two plots to study to the effect of patchiness on population persistence. The population densities in the three plots are represented on Figure $4 \mathrm{~b}$, with a solid, dotted and dashed line corresponding to the $144 \times 144$, checkerboard and $200 \times 200$ plots. 


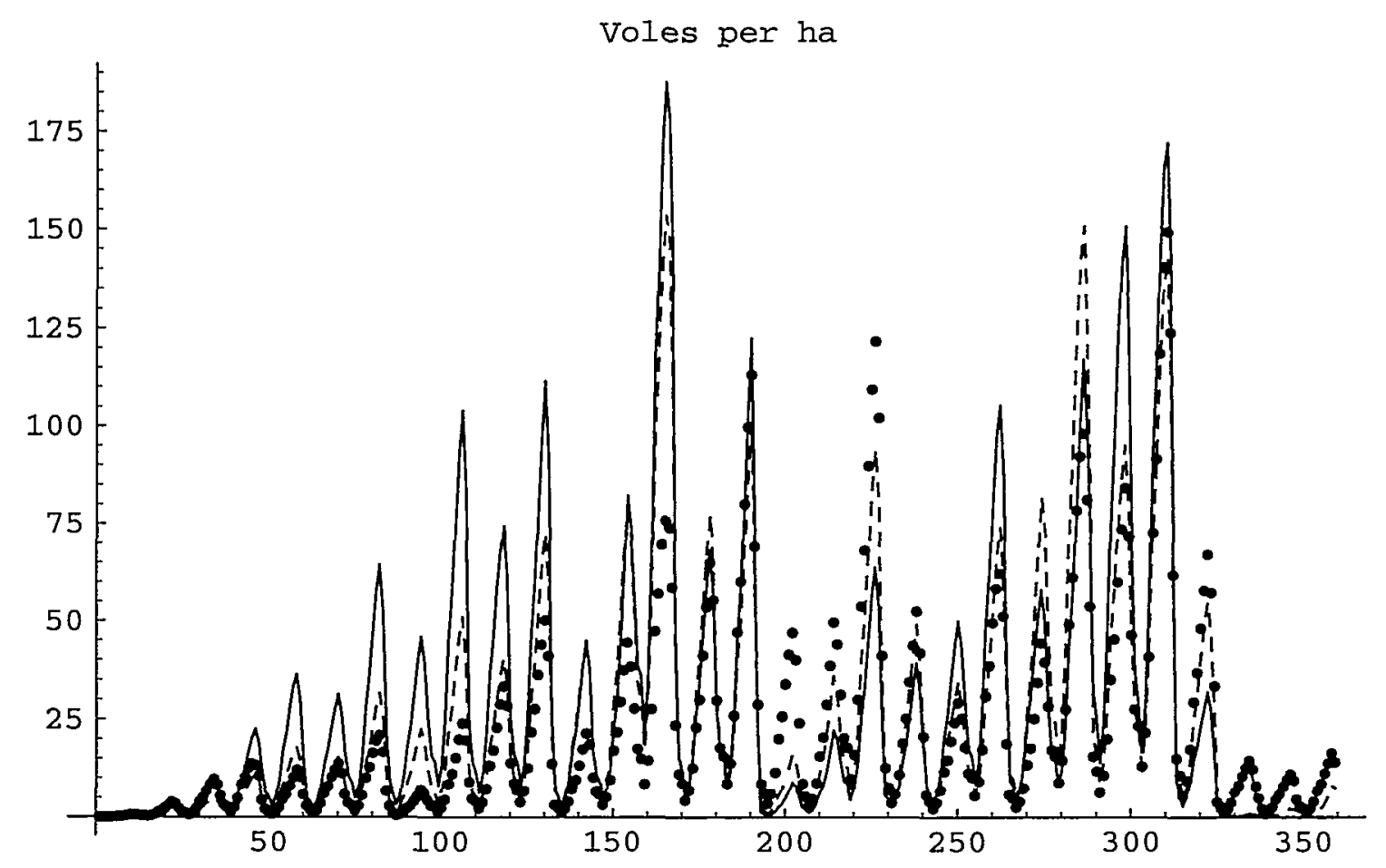

Figure 4b. Comparison of the length of population persistence on three plots of $1866 \mathrm{ha}, 1872 \mathrm{ha}$ and 3600 ha. See text for details.

The population on the $144 \times 144$ plot went extinct near the end of the 30 year period, while the populations in the other two plots persisted till the end.

The maximum population densities per ha were 187, 149 and 153, while the average population densities per ha were $29,21,25$ for the $144 \times 144$, checkerboard and $200 \times 200$ plot respectively. The standart deviation was $7 \%, 6 \%$ and $7 \%$. This simulation shows that the checkerboard fragmentation has, in fact, a beneficial effect on the persistence of the population by reducing the maximum and average density and the amplitude of oscillations.

The results of various simulations with obstacles seem to confirm the conclusion that fragmentation does not have a clearly expressed negative effect on population persistence. Our results show that it is rather the decrease in the size of the inhabited population area that does have a clearly expressed effect on population persistence.

\section{Acknowledgements}

The authors would like to thank Nancy Comstock of the US Department of Energy, National Petroleum Technology Office, for providing financial support through the National Gas and Oil Technology Partnership Program. The authors also thank Yetta Jager and Rebecca Efroymson, Oakridge National Laboratory, for numerous useful discussions. We are indebted to Steve Smith from CASC, LLNL for help with improving the speed of computations and with the visualization of the spatial density distributions, done by using the visualization tool of the SAMRAI package, and to Jessie Coty, ERD, LLNL for help with ecological literature searches.

This work was performed under the auspices of the U.S. Department of Energy by the University of California Lawrence Livermore National Laboratory under contract No. W-7405-Eng-48. 


\section{References}

[1] Abramsky, Z. and C.R. Tracy, (1980), Relation between home range size and regulation of population size in Microtus Ochrogaster. Oikos 34(3): 347-355

[2] De Angelis D.L., L. Gross, M. Huston, W.Wolff, D.M. Fleming, E.J. Cominskey and S. Sylvester, (1998), Landscape modeling for Everglades Ecosystem Restoration, Ecosystems, 1: 64-75

[3] Andren, H. (1994) Effects of habitat fragmentation on birds and mammals in landscapes with different proportions of suitable habitat - a review Oikos 71: 355-366

[4] Baron JS, Ojima DS, Holland EA, Parton WJ, (1994), Analysis of nitrogen saturation potential in rocky-mountain tundra and forest -implications for aquatic systems. Biogeochemistry 27 (1): $61-82$

[5] Bradley, S.R., (1976) Temperature Regulation and Bioenergetics of Some Microtine Rodents, Thesis Cornell University

[6] Carter J. and J. T. Finn, (1999), MOAB: a spatially explicit, individual-based expert system for creating animal foraging models, Ecological Modelling, 1119: 29-41

[7] Chimner RA, Cooper DJ, Parton WJ. (2002), Modeling carbon accumulation in Rocky Mountain fens, Wetlands 22 (1): 100-110.

[8] Cole, F.R. and G.O. Batzli (1979), Nutrition and population dynamics of the prairie vole, Microtus-Ochrogaster in Central Mlinois. Journal of Animal Ecology 48(2):455-470

[9] Conley AH, Holland EA, Seastedt TR, Parton WJ. (2000), Simulation of carbon and nitrogen cycling in on alpine tundra. Arctic Antarctic and Alpine Research 32 (2): 147-154

[10] Desy, E.A. and G.O. Batzli, (1989), Effects of food availability and predation on prairie vole demography: A field experiment. Ecology 70(2):411-421

[11] Fahrih, L. and A.A. Crez (1996) Population spatial structure, human-caused landscape changes and species survival, Revista Chilena de Historia Natural69:5-13

[12] Fahrig, L. (2001) How much habitat is enough? Biological conservation100:65-74

[13] Relative effects of habitat loss and fragmentation on population extinction, Journal of Wildlife management 61: 603-610

[14] Fuks H. and A. Lawniczak, (2001), Individual-based lattice model for spatial spread of epidemics, Discrete Dynamics in Nature and Society, 6(3):191-200

[15] Gaines, M.S. and R.K. Rose, (1976), Population dynamics of Microtus-Ochrogaster in Eastern Kansas. Ecology 57(6):1145-1161

[16] Getz, L.L. and B. McGuire, (1993), A comparison of living singly and in male-female pairs in the prairie vole, Microtus ochrogaster. Ethology 94(4):265-278

[17] Getz, L.L. and J.E. Hofmann, (1999), Diversity and stability of small mammals in tallgrass prairie habitat in central Illinois, USA Oikos 85(2):356-363

[18] Getz, L.L., Simms, L.E. and B. McGuire. (2000), Nestling survival and population cycles in the prairie vole, Microtus ochrogaster. Canadian Journal of Zoology 78(10):1723-1731 
[19] Getz, L.L., Hofmann, J.E., McGuire, B. and T.W. Dolan, (2001). Twenty-five years of population fluctuations of Microtus ochrogaster and M. pennsylvanicus in three habitats in east-central Illinois. Journal of Mammalogy 82(1):22-34

[20] Ginovart, M., D. Lopez and J. Valls, (2002), INDISIM, An individual-based discrete simulation model to study bacterial cultures, Journal of Theoretical Biology, 214: 305-319

[21] Henry R., P.J. Bacon, R. Moss, S.C.F. Palmer and J. McGlade, (1997), A two-dimensional individual-based model of territorial behavior: possible population consequences of kinship in red grouse, Ecological Modelling, 105 : 23-39

[22] Jacquot, J.J. (1999) Use of Space and Movement of Two Vole Species in Response to Habitat Quality. Dissertation, Miami University. 134 pages

[23] W.G.Kelley and A.C.Peterson, (2001) Difference Equations, Harcourt/Academic Press

[24] Krebs C.J., Keller, B.L. and R.H. Tamarin, (1969), Microtus population biology: demographic changes in fluctuating populations of M. ochrogaster and M. pensilvanicus in Southern Indiana. Ecology, 50(4):587-607

[25] Kreft J.U., C. Picioreanu, J.W.T. Wimpenny and MCM van Loosdrecht, (2001), Individualbased modelling of biofilms, Microbiology-SGM, 147: 2897-2912, Part 11

[26] Letcher, B.H., J.A.Priddy, J.R. Walters and L.B.Crowder, (1998), An individual based, spatially explicit simulation model of the population dynamics of the endangered red-cockaded woodpecker, Picoides borealis, Biological Conservation, 86:1-14

[27] McGuire, B. and L.L. Getz, (1998), The nature and frequency of social interactions among freeliving prairie voles (Microtus ochrogaster). Behavioral Ecology and Sociobiology 43(4-5):271-279

[28] McNab, B.K., (1963), Bioenergetics and the determination of the home range size, Am. Naturalist, 97:133-140

[29] Megrey B.A., S. Hinckley and E. Dobbins, (2002), Using scientific visualization tools to facilitate analysis of multi-dimensional data from a spatially-explicit, biophysical, individual-based model of marine fish early life history, ICES Journal of Marine Science, 59: 203-215

[30] Parton W.J., D.S. Schimel, C.V. Cole, D.S. Ojima, (1987), Analysis of factors controlling soil organic-matter levels in great-plains grasslands. Soil Sci. Soc. Am. J 51 (5): 1173-1179.

[31] Parton, W.J., J.W.B. Stewart, C.V.Cole, (1988). Dynamics of c, n, p and s in grassland soils a model. Biogeochemistry 5 (1): 109-131.

[32] Parton WJ, Scurlock JMO, Ojima DS, Gilmanov TG, Scholes RJ, Schimel DS, Kirchner T, Menaut JC, Seastedt T, Moya EG, Kamnalrut A, Kinyamario JI. (1993). Observations and modeling of biomass and soil organic-matter dynamics for the grassland biome worldwide. Global Biogeochem Cy 7 (4): 785-809.

[33] Reuter H. and B. Breckling, (1999), Emerging properties on the individual level: modelling the reproduction phase of the European robin, Ecological Modelling, 121: 199-219

[34] Rose, R.K. and M.S. Gaines (1978) Reproductive cycle of Microtus-Ochrogaster in Eastern Kansas. Ecological Monographs48(1) :21-42 
[35] Sanford RL, Parton WJ, Ojima DS, Lodge DJ. (1991), Hurricane effects on soil organic-matter dynamics and forest production in the luquillo experimental forest, Puerto Rico - results of simulation modeling. Biotropica 23 (4): 364-372 Part A.

[36] Sawicka-Kapusta K., M. Dobrolecka, Drozdz A. and Tertil R., (1975), Bioenergetic parameters of experimental groups of common voles (M. arvalis). Ecologia Polska, 23(2): 347-365

[37] Shnerb, N., Louzoun, Y., Bettelheim,E., Solomon S. (2000) The Importance of Being Discrete - Life Always Wins on the Surface, Proc. Natl. Acad. Sci. USA, 97: 10322;

[38] South, A., (1999), Extrapolating from individual movement behavior to population spacing patterns in a ranging mammal, Ecological Modelling, 117: 343-360

[39] Swihart, R.K. and N.A. Slade, (1989) Differences in Home-Range Size Between Sexes of Microtus-Ochrogaster. Journal of Mammalogy 70(4):941-948

[40] W.F.Wolff, (1994), An individual-oriented model of wading bird nesting colony, Ecological Modelling, 72: 75-114

[41] Ziv, Y., (1998), The effect of habitat heterogeneity on species diversity patterns: a communitylevel approach using an object-oriented landscape simulation model (SHALOM), Ecological Modelling, 111: 135-170

This work was performed under the auspices of the U.S. Department of Energy by the University of California, Lawrence Livermore National Laboratory under Contract No. W-7405-Eng-48. 


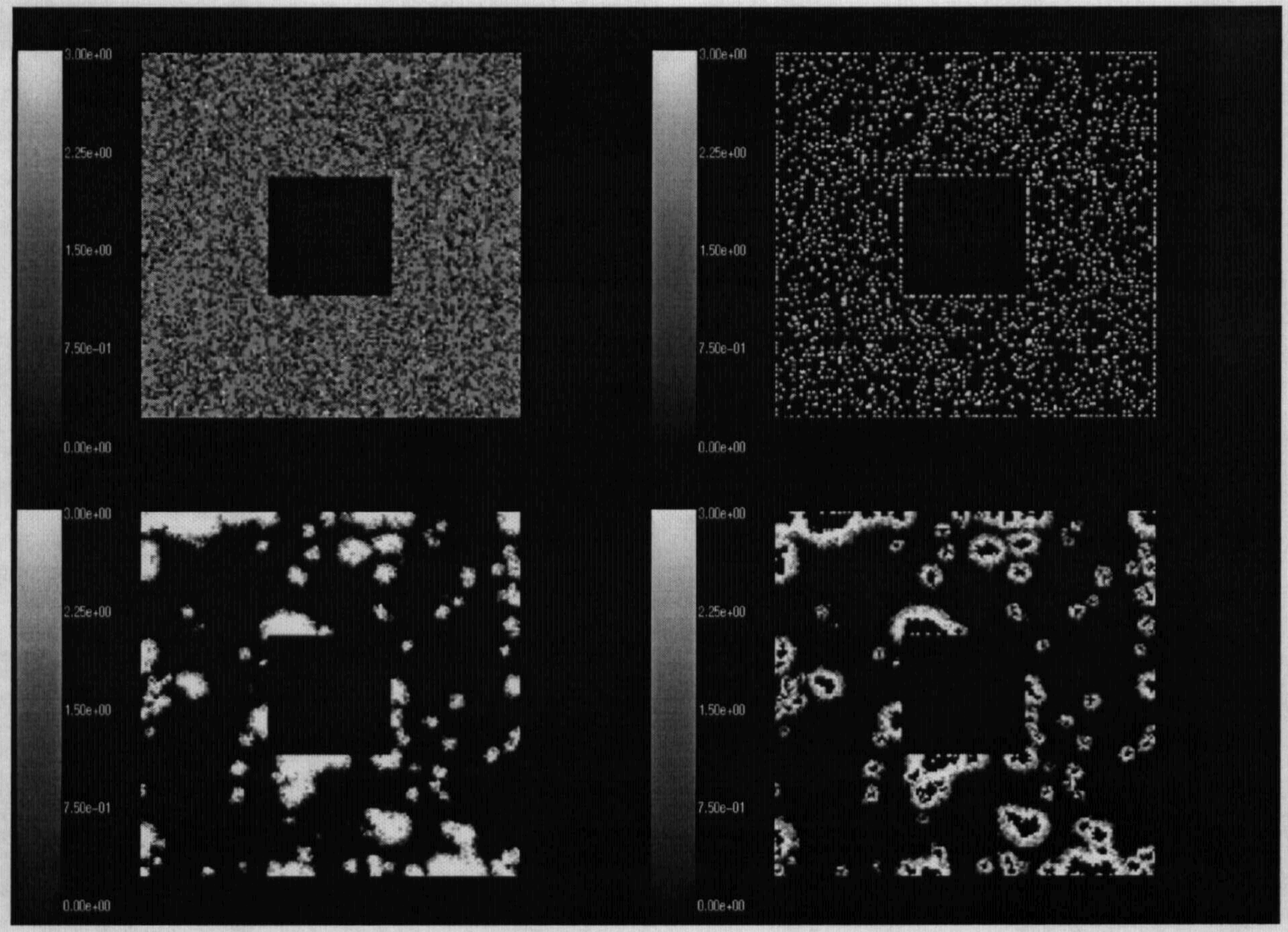

Figure 2. Formation of wave - like patterns from a uniformly random distribution on a spatial plot with a removed central region. The top left graph corresponds to the initial distribution in January of year I, the second on top corresponds to December of year 1, the bottom left - to November of the second year and the bottom right - to December of the second year. 


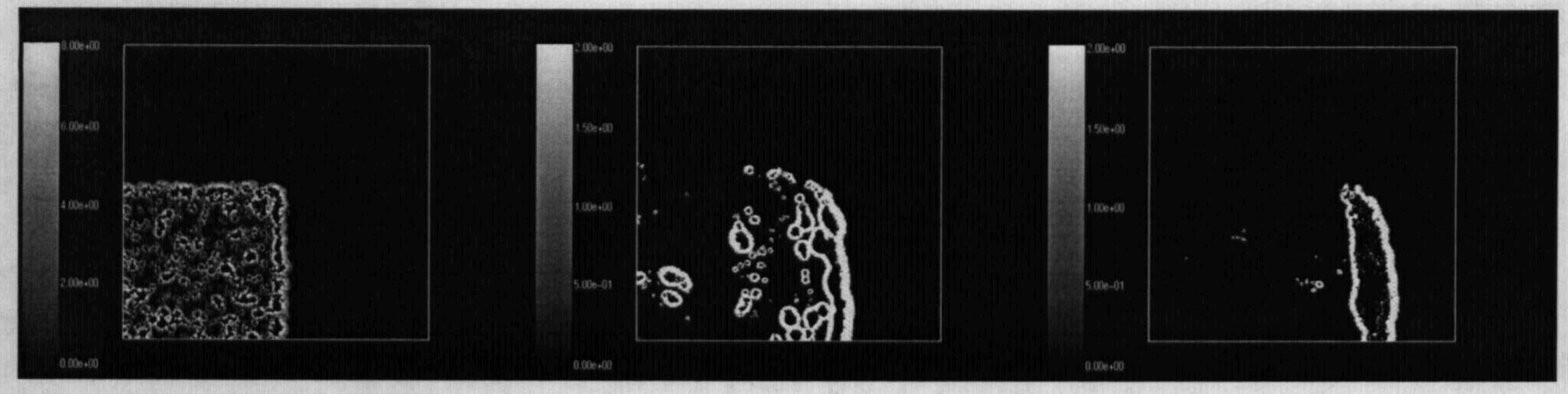

Figure 3a. Spatial distribution of vole densities in a $400 \times 400$ plot in months $24,120,180$ (from left to right). See text. 


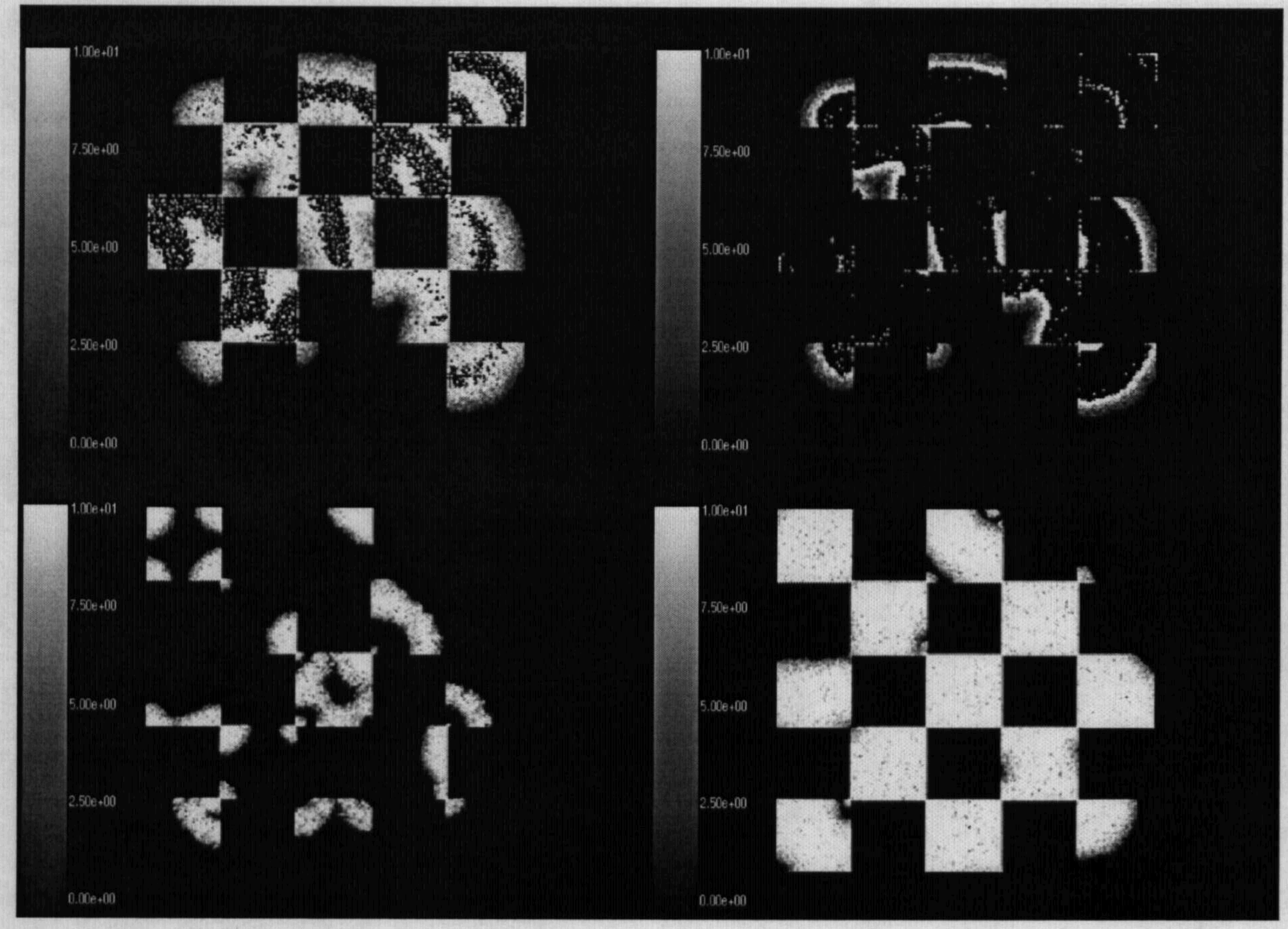

Figure 4a. Density distributions of voles in different points of time in a checkerboard habitat. The overall wave-like progression of density is preserved. 